\title{
Article \\ Identification and Validation of Quantitative Trait Loci Mapping for Spike-Layer Uniformity in Wheat
}

\author{
Kunyu Zhou ${ }^{1,2,+}$, Yu Lin 1,2,+ ${ }^{\text {, Xiaojun Jiang }}{ }^{1,2}$, Wanlin Zhou ${ }^{1,2}$, Fangkun Wu ${ }^{1,2}$, Caixia Li ${ }^{2}$, Yuming Wei ${ }^{1,2}$ \\ and Yaxi Liu 1,2,*(D)
}

check for updates

Citation: Zhou, K.; Lin, Y.; Jiang, X.; Zhou, W.; Wu, F.; Li, C.; Wei, Y.; Liu, Y. Identification and Validation of Quantitative Trait Loci Mapping for Spike-Layer Uniformity in Wheat. Int. J. Mol. Sci. 2022, 23, 1052. https:// doi.org/10.3390/ijms23031052

Academic Editors: Andrés J. Cortés and Hai Du

Received: 19 December 2021

Accepted: 15 January 2022

Published: 19 January 2022

Publisher's Note: MDPI stays neutral with regard to jurisdictional claims in published maps and institutional affiliations.

Copyright: (C) 2022 by the authors. Licensee MDPI, Basel, Switzerland. This article is an open access article distributed under the terms and conditions of the Creative Commons Attribution (CC BY) license (https:// creativecommons.org/licenses/by/ $4.0 /)$
1 State Key Laboratory of Crop Gene Exploration and Utilization in Southwest China, Chengdu 611130, China zhoukunyu@stu.sicau.edu.cn (K.Z.); linyu@stu.sicau.edu.cn (Y.L.); jiangxj999@gmail.com (X.J.); zhouwanlin@stu.sicau.edu.cn (W.Z.); 2018112004@stu.sicau.edu.cn (F.W.); ymwei@sicau.edu.cn (Y.W.)

2 Triticeae Research Institute, Sichuan Agricultural University, Chengdu 611130, China; licaixia@sicau.edu.cn

* Correspondence: liuyaxi@sicau.edu.cn; Tel.: +86-28-86290952; Fax: +86-28-82650350

+ These authors contributed equally to this work.

\begin{abstract}
Spike-layer uniformity (SLU), the consistency of the spike distribution in the vertical space, is an important trait. It directly affects the yield potential and appearance. Revealing the genetic basis of SLU will provide new insights into wheat improvement. To map the SLU-related quantitative trait loci (QTL), 300 recombinant inbred lines (RILs) that were derived from a cross between H461 and Chinese Spring were used in this study. The RILs and parents were tested in fields from two continuous years from two different pilots. Phenotypic analysis showed that H461 was more consistent in the vertical spatial distribution of the spike layer than in Chinese Spring. Based on inclusive composite interval mapping, four QTL were identified for SLU. There were two major QTL on chromosomes 2BL and 2DL and two minor QTL on chromosomes 1BS and 2BL that were identified. The additive effects of QSlu.sicau-1B, Qslu.sicau-2B-2, and QSlu.sicau-2D were all from the parent, H461. The major QTL, QSlu.sicau-2B-2 and QSlu.sicau-2D, were detected in each of the conducted trials. Based on the best linear unbiased prediction values, the two loci explained $23.97 \%$ and $15.98 \%$ of the phenotypic variation, respectively. Compared with previous studies, the two major loci were potentially novel and the two minor loci were overlapped. Based on the kompetitive allele-specific PCR (KASP) marker, the genetic effects for QSlu.sicau-2B-2 were validated in an additional RIL population. The genetic effects ranged from $26.65 \%$ to $32.56 \%$, with an average value of $30.40 \%$. In addition, QSlu.sicau-2B-2 showed a significant $(p<0.01)$ and positive influence on the spike length, spikelet number, and thousand kernel weight. The identified QTL and the developed KASP marker will be helpful for fine-mapping these loci, finally contributing to wheat breeding programs in a marker-assisted selection way.
\end{abstract}

Keywords: QTL mapping; spike-layer uniformity; kompetitive allele-specific PCR marker; yield traits; recombinant inbred lines; wheat

\section{Introduction}

Wheat (Triticum aestivum L.) is one of the most important crops in the world, and it provides $20 \%$ of the global human population with calories [1,2]. To feed the world's growing population, an annual rate increase of $2.4 \%$ in wheat productivity is expected. As the current annual rate of $0.9 \%$ is well below expectations, it is urgent to increase wheat productivity. Breeding high-yield wheat cultivars is one of the most effective strategies to increase the total food production.

Compared with conventional breeding methods, marker-assisted selection is an economic and efficient strategy to breed cultivars. Marker-assisted selection is a tool for breeders, allowing the possibility to select desirable traits more directly using DNA markers that are linked to genes/loci of interest [3]. Quantitative trait loci (QTL) mapping can provide usable molecular markers that are linked to a gene of interest. Up to now, QTL 
mapping has been widely applied to discover the locations of loci underlying traits in plants [4-13]. With the development of high-throughput sequencing technology, single nucleotide polymorphism (SNP) arrays, including the $9 \mathrm{~K}, 55 \mathrm{~K}, 90 \mathrm{~K}, 660 \mathrm{~K}$, and $820 \mathrm{~K}$ arrays, have been successfully used for constructing high-density genetic maps of wheat [14-18]. Based on this, QTL mapping has been used for detection within relatively narrow genetic intervals $[14,15,19]$. The availability of high-throughput SNPs and high-quality reference genomes combine to make the identification of QTL more precise. The identified QTLlinked markers, via dense genetic maps, could be directly used in wheat improvement through molecular assisted selection (MAS) and further accelerating fine mapping and map-based gene cloning.

Spike-layer uniformity (SLU), the consistency of the spike distribution in the vertical space, is determined by the different plant heights $(\mathrm{PH})$ between the main stem and tillers. A uniform spike-layer ensures, not only uniform grain sizes, but also a synchronized maturation time and facilitates harvesting [20]. In rice, ideotype plants with a high yield potential are suggested to have uniformity in the panicle layer [20]. Several genes have been demonstrated to control SLU and affect yield [21,22]. In wheat, the SLU is a quantitative trait and directly affects the yield potential and appearance [23,24]. It has relatively high heritability and is affected by both genotypes and environmental factors [23,24]. A previous study found that the genotype "Jing411" has a higher SLU value than "Kenong9204" [23]. This indicated that "Jing411" has a more consistent vertical spatial distribution. To identify the wheat genotypes with uniform spike-layers and loci that are associated with this trait, a natural population, including 225 diverse bread wheats, was also used in a previous study. In previous studies, QTL for SLU have been identified on chromosomes 1A, 1B, 1D, 2A, 2B, 2D, 3A, 3B, 4A, 4B, 5A, 5B, 6A, 6B, 6D, 7A, and 7D [23,24]. Among these, only one locus, $q S l u-4 A$, was detected in three environments; the others were identified in one or two environments. To breed wheat with consistent spike heights, it is necessary to detect stable QTL of SLU among multiple environments. These expected QTL were reliable in the marker-assisted selection of wheat improvement.

Spike-layer uniformity is an important trait that directly affects wheat yield potential and appearance. Uncovering the genetic basis of SLU in wheat will provide a new insight into wheat improvement. In this study, the SLU of H461 was more consistent than that of Chinese Spring. The derived recombinant inbred line (RIL) population was used to evaluate the SLU from two consecutive years from two different environments. Based on the highdensity map that was constructed by the 55K SNP array, QTL mapping was performed for this trait in multiple environments and using the best linear unbiased prediction (BLUP) values. The genetic effects of the objective locus were validated in a different background using an additional RIL population. Further analysis was performed to reveal the genetic relationships between the SLU and several yield traits.

\section{Results}

\subsection{Phenotypic Characterization of Spike-Layer Uniformity}

A significant $(p<0.01)$ difference was identified for the SLU among genotypes, environments, and genotype-environment interactions (Table 1). In all four trials, the SLU of H461 ranged from 0.66 to 0.83 , while the SLU of Chinese Spring ranged from 0.18 to 0.24 (Figure 1, Table 2). The spike-layer uniformity of H461 was significantly higher than that of Chinese Spring in all four trials (Table 2). Based on the BLUP values, the SLU of H461 was 0.80, which was 3.6 times higher than that of Chinese Spring. The results revealed the great potential of wheat spike-layer improvement using the material $\mathrm{H} 461$. In all the trials, transgressive segregation was observed in the RIL population (Figure 1, Table 2). The broad-sense heritability of SLU was 0.88 , indicating that this trait was majorly controlled by genetic factors. Significant $(p<0.01)$ correlations were observed among all four environments with correlation coefficients ranging from 0.62 to 0.79 (Supplementary Table S1), which indicated that the SLUs that were obtained from the four trials were strongly correlated. 
Table 1. Analysis of variance for spike-layer uniformity across the four environments.

\begin{tabular}{cccccc}
\hline Variable & DF $^{\mathbf{a}}$ & Type III Sum of Square & Mean Square & F Value & Significance $^{\mathbf{b}}$ \\
\hline Environment & 3 & 3.76 & 1.25 & 141.77 & $* *$ \\
Replication & 4 & 0.02 & 0.005 & 0.52 & $\mathrm{~ns}$ \\
Genotype & 298 & 32.51 & 0.11 & 12.35 & $* *$ \\
Environment $\times$ Genotype & 885 & 10.29 & 0.01 & 1.32 & $* *$ \\
\hline
\end{tabular}

${ }^{a}$ DF, degrees of freedom. ${ }^{b * *}$, significant at $p<0.01 ;$ ns, not significant.

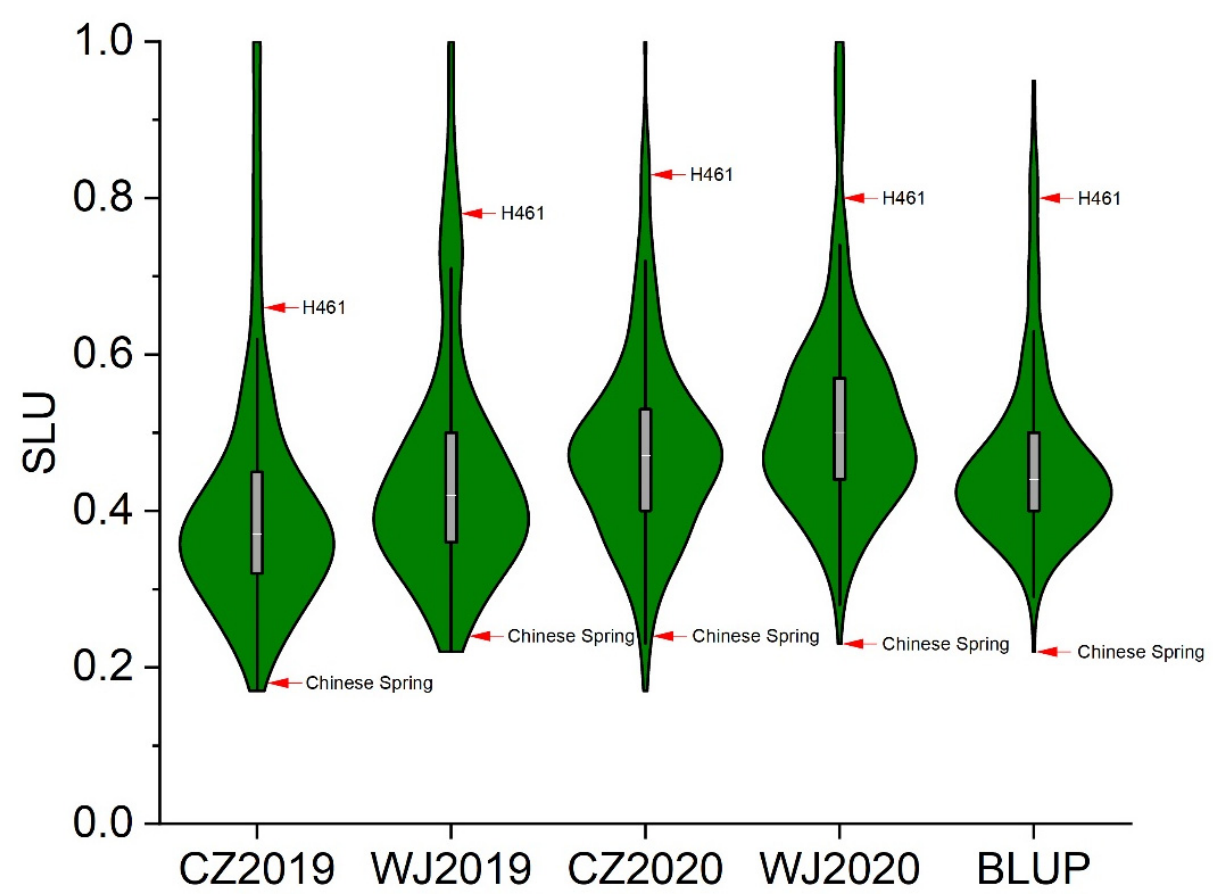

Figure 1. Violin plots for spike-layer uniformity distributions of the $\mathrm{H} 461 \times$ Chinese Spring recombinant inbred line population in different environments. CZ2019, CZ2020, WJ2019, and WJ2020 denote field experiments in Chongzhou $(2019,2020)$ and Wenjiang $(2019,2020)$. BLUP, best linear unbiased prediction.

Table 2. Phenotypic variation and the broad-sense heritability of spike-layer uniformity in different environments.

\begin{tabular}{cccccccccc}
\hline \multirow{2}{*}{ Environment } & \multicolumn{3}{c}{ a } & \multicolumn{5}{c}{ Recombinant Inbred Line (RIL) Population } \\
\cline { 2 - 9 } & H461 & Chinese Spring & Difference $^{\mathbf{b}}$ & Mean & Min & Max & SD $^{\mathbf{c}}$ & CV (\%) $^{\mathbf{d}}$ & Heritability $^{*}$ \\
\hline CZ2019 & 0.66 & 0.18 & $0.49^{* *}$ & 0.41 & 0.17 & 1.00 & 0.15 & 37.61 \\
WJ2019 & 0.78 & 0.24 & $0.53^{* *}$ & 0.45 & 0.22 & 1.00 & 0.15 & 32.84 \\
CZ2020 & 0.83 & 0.24 & $0.59^{* *}$ & 0.48 & 0.17 & 1.00 & 0.12 & 25.11 \\
WJ2020 & 0.80 & 0.23 & $0.57^{* *}$ & 0.52 & 0.28 & 1.00 & 0.13 & 24.34 \\
BLUP & 0.80 & 0.22 & $0.58^{* *}$ & 0.58 & 0.29 & 0.95 & 0.11 & 18.69 \\
\hline
\end{tabular}

${ }^{a}$ CZ2019, CZ2020, WJ2019, and WJ2020 denote field experiments in Chongzhou (2019, 2020) and Wenjiang (2019, 2020). BLUP, best linear unbiased prediction. ${ }^{\mathrm{b}} * *$, significant at $p<0.01 .{ }^{\mathrm{c}} \mathrm{SD}$, standard deviation. ${ }^{\mathrm{d}} \mathrm{CV}$, coefficient of variation.

\subsection{Identification of QTL Conferring Spike-Layer Uniformity}

A total of four QTL were identified for the SLU (Figure 2, Table 3). Among them, two major QTL were located on chromosomes 2B (QSlu.sicau-2B-2) and 2D (QSlu.sicau-2D), and two minor QTL were located on chromosomes 1B (QSlu.sicau-1B) and 2B (QSlu.sicau-2B-1). QSlu.sicau-2B-2 (flanked by AX-108770043 and AX-108927717) was detected in all four trials, as well as in the BLUP values. This locus was located on the long arm of chromosome $2 \mathrm{~B}$ at $165.70-166.40 \mathrm{cM}$ and explained $13.89-23.97 \%$ of the phenotypic variation. The other major locus, QSlu.sicau-2D, was detected in all the trials, as well in the BLUP values. It was located on the long arm of chromosome $2 \mathrm{D}$ at $230.67-235.60 \mathrm{cM}$ and explained 
$12.93-15.98 \%$ of the phenotypic variation. The minor locus QSlu.sicau-1B (flanked by AX109490843 and AX-111619113) was detected in CZ2020, as well as in the BLUP values and was located on the short arm of chromosome 1B at 6.87-7.22 cM, explaining 3.43-3.55\% of the phenotypic variation. The other minor locus, QSlu.sicau-2B-1 (flanked by AX-111487903 and AX-111457622), was detected in WJ2019 as well as in the BLUP values, explaining $2.05-3.91 \%$ of the phenotypic variation. This locus was on the long arm of chromosome $2 \mathrm{~B}$ at 137.91-139.55 cM. With the exception of QSlu.sicau-2B-1, alleles for the higher SLUs at these loci were all from the parent, H461.

1B

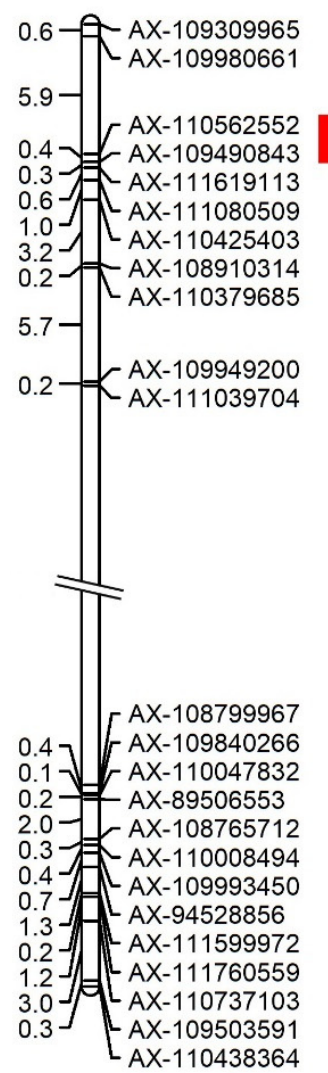

2B

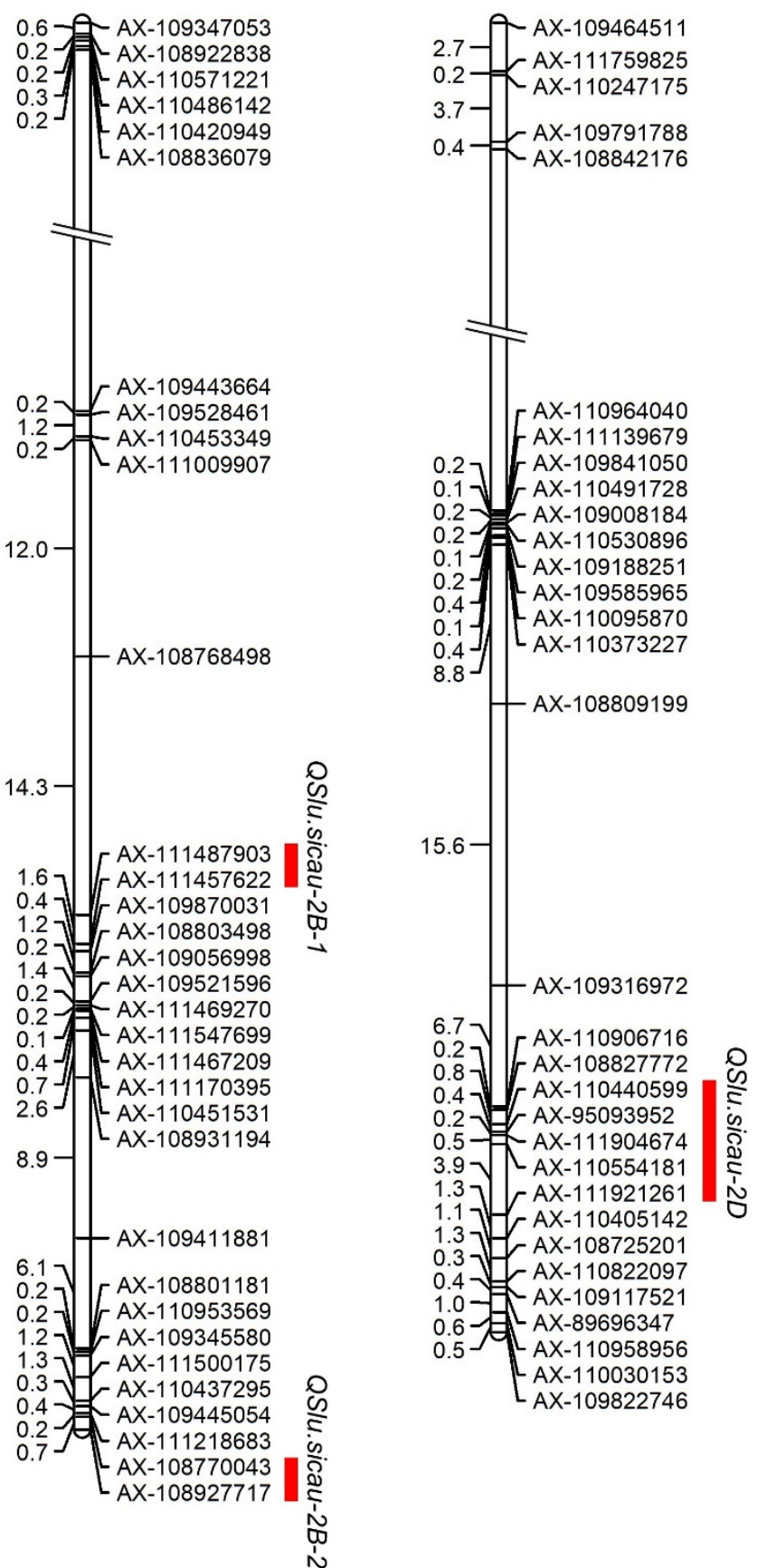

Figure 2. Chromosomal locations of QTL for spike-layer uniformity in the H461 $\times$ Chinese Spring recombinant inbred line population. The red bar points to the genetic interval of QTL. The numbers on the left side of the chromosomes point to the genetic distance (cM) between the markers. The marker's names are shown on the right side of chromosomes. 
Table 3. Quantitative trait loci for spike-layer uniformity that were identified in different environments and using BLUP values.

\begin{tabular}{|c|c|c|c|c|c|c|c|c|}
\hline QTL & Environment $^{a}$ & Chromosome & Genetic Distance (cM) & Physical Positions (Mb) & Flanking Marker & LOD $^{b}$ & $\operatorname{PVE}(\%)^{c}$ & $\mathrm{AE}^{\mathrm{d}}$ \\
\hline \multirow{2}{*}{ QSlu.sicau-1B } & CZ2020 & 1B & $6.87-7.22$ & $572.94-577.58$ & AX-109490843 and AX-111619113 & 3.73 & 3.43 & 0.024 \\
\hline & BLUP & 1B & $6.87-7.22$ & $572.94-577.58$ & AX-109490843 and AX-111619113 & 5.96 & 3.55 & 0.024 \\
\hline QSlu.sicau-2B-1 & WJ2019 & $2 B$ & $137.91-139.55$ & $755.21-777.78$ & AX-111487903 and AX-111457622 & 6.17 & 3.91 & -0.034 \\
\hline \multirow{4}{*}{ QSlu.sicau-2B-2 } & CZ2019 & $2 \mathrm{~B}$ & $165.70-166.40$ & $798.21-802.29$ & AX-108770043 and AX-108927717 & 17.66 & 17.98 & 0.070 \\
\hline & WJ2019 & $2 B$ & $165.70-166.40$ & $788.13-792.19$ & AX-108770043 and AX-108927717 & 28.76 & 21.21 & 0.078 \\
\hline & CZ2020 & $2 B$ & $165.70-166.40$ & $788.13-792.20$ & AX-108770043 and AX-108927717 & 14.06 & 13.89 & 0.048 \\
\hline & WJ2020 & $2 \mathrm{~B}$ & $165.70-166.40$ & $788.13-792.21$ & AX-108770043 and AX-108927717 & 16.12 & 16.73 & 0.055 \\
\hline \multirow{5}{*}{ QSlu.sicau-2D } & CZ2019 & $2 \mathrm{D}$ & $231.72-235.60$ & $565.41-640.84$ & AX-110554181 and AX-111921261 & 13.61 & 13.67 & 0.062 \\
\hline & WJ2019 & $2 \mathrm{D}$ & 230.67-231.02 & $633.66-634.41$ & AX-110440599 and AX-95093952 & 18.98 & 12.93 & 0.061 \\
\hline & CZ2020 & $2 \mathrm{D}$ & $231.72-235.60$ & $565.41-640.84$ & AX-110554181 and AX-111921261 & 14.46 & 14.88 & 0.050 \\
\hline & WJ2020 & $2 \mathrm{D}$ & $231.72-235.60$ & $565.41-640.84$ & AX-110554181 and AX-111921261 & 14.84 & 15.39 & 0.053 \\
\hline & BLUP & $2 \mathrm{D}$ & 230.67-231.02 & $633.66-634.41$ & AX-110440599 and AX-95093952 & 23.75 & 15.98 & 0.051 \\
\hline
\end{tabular}

${ }^{a}$ CZ2019, CZ2020, WJ2019, and WJ2020 denote field experiments in Chongzhou $(2019,2020)$ and Wenjiang $(2019,2020)$. BLUP, best linear unbiased prediction. ${ }^{b}$ LOD, the logarithm of odds score. ${ }^{c}$ PVE, the percentage of phenotypic variance explained by individual QTL. ${ }^{d}$ AE, additive effect. Positive values mean alleles from H461 increase the trait scores, and negative values mean alleles from Chinese Spring increase the trait scores. 
2.3. Validation of the Major and Stable QTL, with a Different Genetic Background and Its Effect on Yield-Related Traits

The tightly linked SNP, AX-108927717, was converted into a kompetitive allele-specific PCR (KASP) marker (KASP-7717) to validate the major and stable locus, QSlu.sicau-2B-2, with a different genetic background (Table 4). The KASP marker was polymorphic, between H461 and the other two parents (Chinese Spring and CM42). In the RILs that were derived from H461 and CM42, the SLU of the group carrying the "aa" allele was significantly $(p<0.01)$ higher than that of the group carrying the "AA" allele (Figure 3$)$. The differences between the two groups were $32.56 \%, 31.98 \%$, and $26.65 \%$ for CZ2019, WJ2019, and BLUP, respectively, with an average value of $30.40 \%$ (Figure 3 ). In addition, the spike length (SL) of the group carrying the "aa" allele was significantly $(p<0.05)$ higher than that of the group carrying the "AA" allele. The group carrying the "aa" allele had a significantly $(p<0.01)$ higher spikelet number (SN) and thousand kernel weight (TKW) than the group carrying the "AA" allele. Thus, QSlu.sicau-2B-2 showed a significant and positive influence on SL, SN, and TKW, but it did not affect PH (Table 5). These results indicated that this locus has great potential in wheat yield improvement.

Table 4. The primers of the kompetitive allele-specific marker for alleles of Qslu.sicau-2B-2 that were used in this study.

\begin{tabular}{cccc}
\hline SNP & Primer $^{\text {a }}$ & Primer sequence (5' to $\mathbf{3}^{\prime}$ ) & Allele \\
\hline \multirow{3}{*}{ AX-108927717 } & FAM & TTGGAATGTCTCCATCCCAC & aa \\
& HEX & TTGGAATGTCTCCATCCCAG & AA \\
& Common reverse & CCTCTCCTATATCTGGCTTCTGTTG & \\
\hline
\end{tabular}

a FAM probe sequence of the forward primer is GAAGGTGACCAAGTTCATGCT, and the HEX probe sequence of the reverse primer is GAAGGTCGGAGTCAACGGATT. $\mathbf{b}$ aa, lines carrying the homozygous alleles from H461; AA, lines carrying the homozygous alleles from the non- $\mathrm{H} 461$ parents.

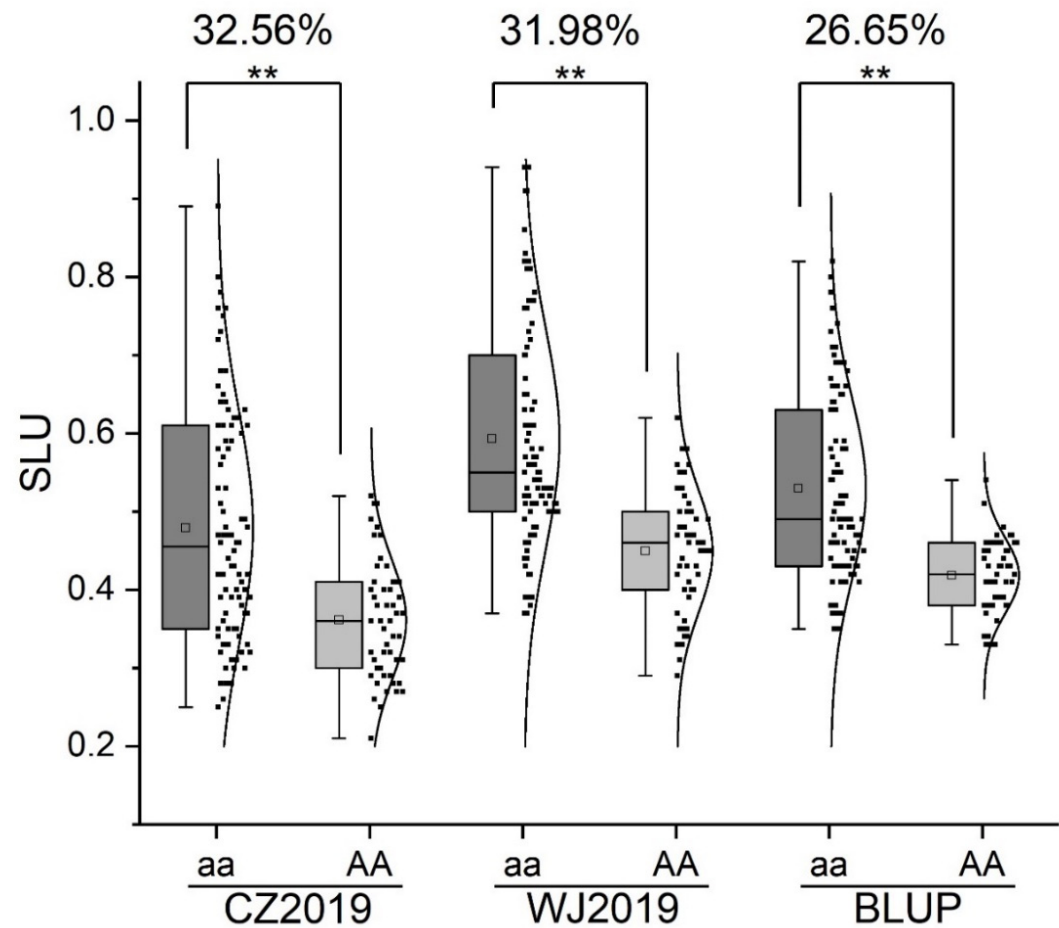

Figure 3. Effects of Qslu.sicau-2B-2 in the H461 × CM42 recombinant inbred line population. Aa, lines carrying the homozygous alleles from H461. AA, lines carrying the homozygous alleles from the non-H461 parents. CZ2019 and WJ2019 denote the field experiments in 2019 in Chongzhou and Wenjiang, respectively. BLUP, best linear unbiased prediction. ${ }^{* *}$, significant at $p<0.01$. 
Table 5. Effects of QSlu.sicau-2B-2 on the yield-related traits in the $\mathrm{H} 461 \times \mathrm{HC} 42$ recombinant inbred line population.

\begin{tabular}{cccccc}
\hline Environment $^{\mathbf{a}}$ & Allele & $\begin{array}{c}\text { Plant Height } \\
\mathbf{( c m )}\end{array}$ & $\begin{array}{c}\text { Spike Length } \\
(\mathbf{c m})^{\mathbf{b}}\end{array}$ & $\begin{array}{c}\text { Spikelet } \\
\text { Number }\end{array}$ & $\begin{array}{c}\text { Thousand Kernel } \\
\text { Weight } \mathbf{( g )}\end{array}$ \\
\hline \multirow{2}{*}{ CZ2019 } & aa & 84.22 & $13.60^{*}$ & $22.11^{* *}$ & $50.15^{* *}$ \\
& AA & 84.22 & 13.23 & 20.81 & 45.84 \\
WJ2019 & aa & 82.01 & $13.45^{* *}$ & $20.92^{* *}$ & $50.15^{* *}$ \\
& AA & 82.01 & 12.80 & 19.74 & 45.84 \\
BLUP & aa & 83.10 & $13.50^{*}$ & $21.48^{* *}$ & $47.58^{* *}$ \\
& AA & 81.25 & 13.05 & 20.35 & 44.60 \\
\hline
\end{tabular}

${ }^{a}$ CZ2019 and WJ2019 denote field experiments in 2019 in Chongzhou and Wenjiang, respectively. BLUP, best linear unbiased prediction. ${ }^{\mathrm{b}} *$, significant at $p<0.05 .{ }^{* *}$, significant at $p<0.01$.

\subsection{Candidate Genes for QSlu.sicau-2B-2}

Based on the Chinese Spring Reference Genome Sequence RefSeq v2.1 [25,26], QSlu.sicau$2 B-2$ was located on the long arm of chromosome $2 \mathrm{~B}$ at 798.21-802.29 Mb. A total of 49 predicted genes were identified in the interval. After homologous analysis, a total of 47 genes were homologous with rice. There were two homologous genes of rice that have been reported regarding their functions, GSTU5 [27] and GSTU45 [27]. Expression analysis of these genes is shown in Figure S1. TraesCS2B03G1529700, which encodes thioredoxin, showed the highest expression, from the seedling stage to the dough stage (Table S2). The expressions of eight genes, including TraesCS2B03G1522100, TraesCS2B03G1522700, TraesCS2B03G1524300, TraesCS2B03G1526400, TraesCS2B03G1531500, TraesCS2B03G1531600, and TraesCS2B03G1534400, were shown to be highly expressed in all developmental stages.

\section{Discussion}

The uniformity of the spike layer significantly affects the plant architecture and grain yield in wheat. The high heritability of SLU in the present study indicated that genetic factors strongly determined this trait (Table 1). The high heritability for SLU was also observed in the population that included 20 wheat cultivars/lines [28]. Previous studies also confirmed that genetic factors mainly controlled SLU $[22,28,29]$. Thus, it is necessary to understand the genetic basis of SLU for MAS to be applied in wheat breeding.

The genetic basis of SLU is poorly understood in wheat. In rice, a few genes have been demonstrated to control plant uniformity, including DWT1, DWL2, and OsPIP5K1 [21,22]. DWT1 encodes a WOX transcription factor and interacts with OsPIP5K1 to coordinate rice uniformity [21,22]. DWL2 has the same redundant functions as DWT1 and acts with OsPIP5K1 to coordinate uniformity in rice [21]. To the best of our knowledge, only two studies on QTL identification for SLU have been reported [23,24]. A total of 19 QTL have been detected and are located on chromosomes 1B, 1D, 2A, 2B, 2D, 4A, 4B, 5A, 5B, 6B, 7A, and 7D. Based on the RIL population consisting of 188 lines, 16 QTL were detected on chromosomes 1B, 1D, 2A (2 QTL), 2B (2 QTL), 4A, 4B, 5A, 5B, 6B (2 QTL), 7A (2 QTL), and 7D (2 QTL), explaining $5.04-18.43 \%$ phenotypic variation. Of those, one ( $q S \operatorname{Sl}-4 A)$ could be detected across three environments and two ( $q S l u-4 B$ and $q S l u-6 B .1)$ across two environments [23]. In the present study, only four QTL were detected for SLU (Figure 2, Table 3). This is due to the loci that were detected in only one environment being removed in this study. It is well known that QTL that are detected in one environment are not useful in breeding. Due to the significant differences between parents, the large population, and abundant polymorphism markers, two stable and major QTL were detected in this study. Recently, using a set of 225 diverse spring wheat accessions, 14 marker-trait associations for SLU were detected on chromosomes 1A, 1B, 2D, 3A, 3B, 4A, 4B, 5A, 5B, 6A, 6B, 6D, and 7A [24]. Only the SNP, SNP_10924, was significantly associated with the SLU in two environments, explaining up to $6 \%$ of the phenotypic variation [24]. These results indicated that detecting a more stable and major QTL for SLU is necessary.

Based on the Chinese Spring Reference Sequence RefSeq v2.1 [25,26], QTL were compared with previous studies [23,24]. In this study, one minor-effect QTL, QSlu.sicau-1B, 
was identified on chromosome $1 \mathrm{~B}$ at $572.94-577.58 \mathrm{Mb}$. In a previous study, the marker SNP_11184 was located on the short arm of chromosome 1B and was significantly associated with SLU [24]; it was far from QSlu.sicau-1B. In another study, the locus $q S l u-1 B L$ was identified on chromosome 1B in the interval between wPt-2315 and AX-108850061 [23]. Due to the unavailable physical position of the DArT marker wPt-2315, the physical position of this marker was referred to by the adjacent marker, AX-111595814. Thus, $q S l u-1 B L$ is probably located on chromosome $1 \mathrm{~B}$ at $575.35-587.26 \mathrm{Mb}$, overlapping with QSlu.sicau-1B. QSlu.sicau-2B-1 was another minor-effect QTL. This QTL was located on chromosome 2B at 755.21-777.78 Mb, overlapping with qSlu-2B.2 (flanked by AX-111041164 and AX-94403958) [23]. The locus $q S l u-2 B .2$ was located on chromosome $2 B$ at 735.06-779.53 Mb. QSlu.sicau-2B-2 was a major and stable QTL with the highest effect. This QTL was located on the long arm of chromosome $2 \mathrm{~B}$ at 798.21-802.29 Mb. It is far from qSlu-2B.1 (chromosome $2 \mathrm{~B}$ at 725.48-725.76 Mb) and $q S l u-2 B .2$ (chromosome 2B at 735.06-779.53 Mb), indicating that QSlu.sicau-2B-2 is a potential novel QTL for SLU. QSlu.sicau-2D is another QTL with a major effect. This QTL is located on the long arm of chromosome 2D at 565.41-640.84 Mb. One of the SLU-related QTL on the short arm of chromosome 2D was reported previously [24], indicating QSlu.sicau-2D as another potential novel QTL. A tightly linked marker of QSlu.sicau-2B-2 was designed to be a KASP marker that was applied in wheat molecular breeding programs (Table 4). The KASP marker, KASP-7717, was genotyped in the HC42 population. The locus QSlu.sicau-2B-2 showed a great effect in a different background with an average difference of $30.40 \%$ (Figure 3 ). This result indicated that QSlu.sicau-2B-2 in MAS for plant architecture improvement in wheat is practicable.

Spike-layer uniformity is a vital plant architecture trait and affects the yield potential in crops with tillers, such as rice, barley, and wheat. Previous studies have found that the SLU and yield potential were positively correlated [22,29-31]. It has been demonstrated that DWT1 regulates intra-panicle uniformity and coordinates panicle and internodes in rice [22]. In wheat, knowledge of the genetic relationships between the SLU and yieldrelated traits is limited. A previous study found that the SLU was significantly negatively correlated with PH, TKW, and the yield per plant [23]. In the present study, QSlu.sicau-2B-1, located on chromosome 2B at 755.21-777.78 Mb, was overlapped with the interval of QSl.sicau-2B-1 (Tables 3 and S3). The additive effects of these two QTL were both from Chinese Spring. This indicated that this locus could regulate both the SLU and SL and not the lowest tiller height (LTH) or PH. QSlu.sicau-2B-2, located on chromosome 2B at 798.21-802.29 Mb, was overlapped with the interval of QGns.sicau-2B [14]. This locus was also overlapped with the interval of QSl.sicau-2B-2 (Tables 3 and S3). The additive effects of these three QTL were both from H461. This indicated that this locus could regulate SLU and coordinate the grain number per spikelet and SL. In the RIL population that was derived from the crossing of $\mathrm{H} 461$ and $\mathrm{CM} 42$, QSlu.sicau-2B-2 also had a positive influence on SL, SN, and TKW, but did not affect PH (Table 5). This finding was inconsistent with previous results [23] indicating that the mechanism of QSlu.sicau-2B-2 may be different. This locus could improve the uniformity of the spike layer and several yield-related traits, demonstrating that applications of QSlu.sicau-2B-2 in wheat yield improvement by MAS are feasible and necessary. A total of 49 genes were located in the interval of QSlu.sicau-2B-2. Of those, eight showed high expression in the wheat development stages (from the seedling stage to the dough stage, Figure S1 and Table S2). TraesCS2B03G1529700 encodes chaperone protein DnaJ (Table S2). A previous study also found that the gene that encodes chaperone protein DnaJ was associated with wheat spike development, seed development, and the grain yield [24]. Combined with expression analyses, TraesCS2B03G1529700 may be the most critical candidate gene for this locus. To validate it, qRT-PCR and transgene testing should be conducted in further studies. 


\section{Materials and Methods}

\subsection{Plant Materials, Trial Design, and Phenotype Evaluation}

A total of 300 RILs $\left(\mathrm{F}_{8}\right)$ that were derived from the cross between H461, and Chinese Spring (denoted as the HCS population) were used to map the SLU-related QTL in this study [14]. In addition, 200 RILs $\left(\mathrm{F}_{8}\right)$ that were derived from the crossing of H461 and CM42 (denoted as the HC42 population) were used to validate the genetic effects of the major QTL. The SLU of H461 was significantly higher than that of Chinese Spring and CM42.

The HCS population was evaluated in Chongzhou $\left(30^{\circ} 32^{\prime} \mathrm{N}, 103^{\circ} 38^{\prime} \mathrm{E}\right)$ in 2019 (CZ2019) and 2020 (CZ2020), and in Wenjiang (30 $\left.43^{\prime} \mathrm{N}, 103^{\circ} 51^{\prime} \mathrm{E}\right)$ in 2019 (WJ2019) and 2020 (WJ2020). The HC42 population was evaluated in CZ2019 and WJ2019. Chongzhou and Wenjiang have yellow-brown soil and paddy soil, respectively. The plant dates and meteorological data, including the temperature and rainfall, of these four trials are presented in Table S4. The experiment was set as an incomplete block design with two repetitions for each environment. Each line contained 15 plants that were grown in a single row for each repetition. The row length was $150 \mathrm{~cm}$ and the row spacing was $30 \mathrm{~cm}$. Standard irrigations, fertilization management, and pest and disease preventions were performed throughout the developmental period. The lowest tiller height (LTH), PH, and SL of the HCS population were evaluated in all four environments. The LTH, PH, SL, SN, and TKW of the HC42 population were also assessed. Each plant's lowest and highest productive tiller was used to evaluate the LTH and PH, respectively. The LTH and PH were measured from the ground level to the tip of the spike, excluding awns. The SL was measured from the base to the tip of the spike, excluding awns. The SN was counted as the number of fertile spikelets per spike. TKW was measured as twice the weight of 500 kernels, harvested in the middle row at the maturation stage. The SLU was calculated as SL/(PH-LTH+SL), as shown in Figure S2.

\subsection{Phenotypic Data Analysis}

The average values of each conducted trial were calculated using SPSS 20.0 (IBM Corp., Armonk, NY, USA). The BLUP values were calculated across all the conducted trials using SAS 9.4 (SAS Institute Inc., Cary, NC, USA), as reported previously [32,33]. The broad-sense heritability of SLU was calculated as $H^{2}=\mathrm{Vg} /(\mathrm{Vg}+\mathrm{Vge} / \mathrm{n}+\mathrm{Ve} / \mathrm{nr})$; where, $\mathrm{Vg}$, Vge, and $\mathrm{Vg}$ are the estimates of variances of genotype, genotype $\times$ environment interaction, and random error variances, respectively, while $n$ and $r$ are the number of environments and replicates [34]. The Student's $t$-test was used to evaluate the SLU differences between H461 and Chinese Spring.

\subsection{Genotyping, Map Construction, QTL Mapping}

The HCS population was genotyped using the wheat 55K SNP array containing 53,063 markers from our previous study [14]. The high-density linkage map of the HCS population contained 21,197 SNPs (represented by 3087 bin markers), spanning $3792.71 \mathrm{cM}$ in total, as described in our previous study [14]. The linkage map contained 21 groups corresponding to 21 wheat chromosomes, respectively. QTL mapping for SLU was detected using the inclusive composite interval mapping [35] in IciMapping 4.2 [36]. To increase the authenticity and reliability of the QTL detected, the threshold was set as LOD scores $\geq 3$, and the loci that were detected in only one environment or only using BLUP values were removed [37-39]. QTL were named according to the rules that were established by the International Rules of Genetic Nomenclature (http:/ / wheat.pw.usda.gov/ggpages/wgc/ 98/Intro.htm (accessed on 12 September 2021)). For example, "Slu" and "sicau" represent "spike-layer uniformity" and "Sichuan Agricultural University," respectively. The flanking markers of the QTL were mapped on the Chinese Spring Reference Sequence RefSeq v2.1 to obtain their chromosomal and physical locations $[25,26]$.

\subsection{Marker Development, QTL Validation, and Candidate Genes Prediction}

SNP probe sequences flanking the target QTL were used to convert them into KASP markers. KASP primers were designed and analyzed following the procedure that was 
described by Suzuki et al. (1991), as in our previous report [40,41]. The amplification reactions were performed in a total volume of $10 \mu \mathrm{L}$, containing $5 \mu \mathrm{L}$ of $2 \times$ KASP Mastermix (JasonGen, Beijing, China), $1.4 \mu \mathrm{L}$ of KASP Assay Mix (mixture of $0.168 \mu \mathrm{mol}$ of FAM primer, $0.168 \mu \mathrm{mol}$ of HEX primer, and $0.42 \mu \mathrm{mol}$ common reverse primer), $2.6 \mu \mathrm{L}$ of DNase/RNase-free water, and $1 \mu \mathrm{L}$ of genomic DNA (concentration of $\sim 100 \mathrm{ng} / \mu \mathrm{L}$ ). The amplifications were carried out using the CFX96 Touch ${ }^{\mathrm{TM}}$ real-time PCR detection system (BioRad, Hercules, California, USA) with the following steps: $95^{\circ} \mathrm{C}$ for $15 \mathrm{~min}$, 10 touchdown cycles for $65-55^{\circ} \mathrm{C}$ (decreasing by $1{ }^{\circ} \mathrm{C}$ per cycle) for $60 \mathrm{~s}$, and 30 cycles for $94{ }^{\circ} \mathrm{C}$ for $20 \mathrm{~s}$ and $57^{\circ} \mathrm{C}$ for $60 \mathrm{~s}$.

For the HCM population, 141 randomly selected lines were genotyped using the designed KASP marker. The RILs were classed into two groups based on the alleles that were present in the parents and their lines. The lines carrying homozygous alleles from H461 and non-H461 parents were denoted as "aa" and "AA", respectively. The Student's $t$-test was used to calculate the significances of the traits between the two groups. Based on Chinese Spring Reference Sequence RefSeq v2.1, the predicted genes that were located in the physical interval of the QTL were determined using homologous analysis by KOBAS 3.0 [42] and funRiceGenes [43] using rice (Oryza sativa) as the background species. In silico gene expression analysis was also performed to identify candidate genes from the Wheat Expression Browser (http:/ / www.wheat-expression.com/ (accessed on 7 January 2022)).

\section{Conclusions}

The wheat line H461 was more consistent in the vertical spatial distribution of the spike layer than Chinese Spring. The spike-layer uniformity of 300 RILs that were derived from these two parents was evaluated in multiple environments. Combined with a previously constructed high-density genetic map, two major-effect QTL were successfully identified on the long arms of chromosomes $2 \mathrm{~B}$ and 2D, respectively. There were two minor-effect QTL that were identified on the short arm of chromosome $1 \mathrm{~B}$ and the long arm of chromosome 2B. A comparison analysis that was based on a physical map of Chinese Spring indicated that the two major loci, QSlu.sicau-2B-2 and QSlu.sicau-2D, were probably novel. The effect of QSlu.sicau-2B-2 was validated in an additional RIL population using a developed KASP marker. QSlu.sicau-2B-2 also positively affected SL, SN, and TKW. The results indicated that this QTL and KASP marker could be used in MAS for new wheat cultivar breeding with a scientifically reasonable spike-layer distribution.

Supplementary Materials: The following are available online at https:/ /www.mdpi.com/article/10 .3390/ijms23031052/s1.

Author Contributions: Conceptualization, Y.L. (Yaxi Liu).; methodology, K.Z. and Y.L. (Yu Lin); software, Y.L. (Yu Lin); validation, K.Z. and X.J.; formal analysis, K.Z., Y.L. (Yu Lin) and X.J.; investigation, W.Z., F.W. and C.L.; resources, Y.L. (Yaxi Liu); data curation, C.L. and Y.L. (Yaxi Liu); writing-original draft preparation, K.Z., Y.L. (Yu Lin) and X.J.; writing-review and editing, Y.L. (Yu Lin), Y.W. and Y.L. (Yaxi Liu); visualization, Y.L. (Yu Lin); supervision, Y.W. and Y.L. (Yaxi Liu); project administration, C.L.; funding acquisition, Y.L. (Yaxi Liu). All authors have read and agreed to the published version of the manuscript.

Funding: This study was funded by the Key Research and Development Program of Sichuan Province (2021YFN0034 and 2021YFG0028) and the National Natural Science Foundation of China (31771794).

Institutional Review Board Statement: Not applicable.

Informed Consent Statement: Not applicable.

Data Availability Statement: The data presented in this study are available on request from the corresponding author.

Conflicts of Interest: The authors declare no conflict of interest. 


\section{References}

1. Chaves, M.S.; Martinelli, J.A.; Wesp-Guterres, C.; Graichen, F.A.S.; Brammer, S.P.; Scagliusi, S.M.; Da Silva, P.R.; Wiethoelter, P.; Montan Torres, G.A.; Lau, E.Y.; et al. The importance for food security of maintaining rust resistance in wheat. Food Secur. 2013, 5, 157-176. [CrossRef]

2. Sinchire, D.B.M.; Álvarez, L.S.J.; Valdivieso, J.I.B.; Mora, E.D.C. Oat and wheat forage production under hydroponic and conventional systems. Cienc. Tecnol. Agropecu. 2020, 21,1-16. [CrossRef]

3. Henkrar, F.; Udupa, S. Marker assisted selection in plant breeding. Moroccan J. Agric. Sci. 2020, 1, $237-247$.

4. Hu, X.; Wang, G.; Du, X.; Zhang, H.; Xu, Z.; Wang, J.; Chen, G.; Wang, B.; Li, X.; Chen, X. QTL analysis across multiple environments reveals promising chromosome regions associated with yield-related traits in maize under drought conditions. Crop J. 2020, 9, 759-766. [CrossRef]

5. Wang, Z.; Hu, H.; Jiang, X.; Tao, Y.; Lin, Y.; Wu, F.; Hou, S.; Liu, S.; Li, C.; Chen, G. Identification and validation of a novel major quantitative trait locus for plant height in common wheat (Triticum aestivum L.). Front Genet. 2020, 11, 1314. [CrossRef]

6. Zhao, M.; Ma, Z.; Wang, L.; Tang, Z.; Mao, T.; Liang, C.; Gao, H.; Zhang, L.; He, N.; Fu, L. SNP-based QTL mapping for panicle traits in the japonica super rice cultivar Liaoxing 1. Crop J. 2020, 8, 769-780. [CrossRef]

7. Zhou, H.; Luo, W.; Gao, S.; Ma, J.; Zhou, M.; Wei, Y.; Zheng, Y.; Liu, Y.; Liu, C. Identification of loci and candidate genes controlling kernel weight in barley based on a population for which whole genome assemblies are available for both parents. Crop J. 2020, 9 , 854-861. [CrossRef]

8. Zhu, X.; Leiser, W.L.; Hahn, V.; Würschum, T. Identification of seed protein and oil related QTL in 944 RILs from a diallel of early-maturing European soybean. Crop J. 2020, 9, 238-247. [CrossRef]

9. Song, J.; Li, B.; Cui, Y.; Zhuo, C.; Gu, Y.; Hu, K.; Wen, J.; Yi, B.; Shen, J.; Ma, C.; et al. QTL mapping and diurnal transcriptome analysis identify candidate genes regulating brassica napus flowering time. Int. J. Mol. Sci. 2021, 22, 7559. [CrossRef] [PubMed]

10. He, J.; Zhang, D.; Chen, X.; Li, Y.; Hu, M.; Sun, S.; Su, Q.; Su, Y.; Li, S. Identification of QTLs and a candidate gene for reducing pre-harvest sprouting in Aegilops tauschii-Triticum aestivum chromosome segment substitution lines. Int. J. Mol. Sci. 2021, 22, 3729. [CrossRef] [PubMed]

11. Arif, M.A.R.; Attaria, F.; Shokat, S.; Akram, S.; Waheed, M.Q.; Arif, A.; Boenier, A. Mapping of QTLs associated with yield and yield related traits in durum wheat (Triticum durum Desf.) under irrigated and drought conditions. Int. J. Mol. Sci. 2020, 21, 2372. [CrossRef] [PubMed]

12. Talukder, Z.I.; Underwood, W.; Ma, G.; Seiler, G.J.; Misar, C.G.; Cai, X.; Qi, L. Genetic dissection of phomopsis stem canker resistance in cultivated sunflower using high density SNP linkage map. Int. J. Mol. Sci. 2020, 21, 1497. [CrossRef] [PubMed]

13. Hina, A.; Cao, Y.; Song, S.; Li, S.; Sharmin, R.A.; Elattar, M.A.; Bhat, J.A.; Zhao, T. High-resolution mapping in two ril populations refines major "QTL hotspot" regions for seed size and shape in soybean (Glycine max L.). Int. J. Mol. Sci. 2020, 21, 1040. [CrossRef] [PubMed]

14. Lin, Y.; Jiang, X.; Hu, H.; Zhou, K.; Wang, Q.; Yu, S.; Yang, X.; Wang, Z.; Wu, F.; Liu, S. QTL mapping for grain number per spikelet in wheat using a high-density genetic map. Crop J. 2021, 9, 1108-1114. [CrossRef]

15. Cui, F.; Zhang, N.; Fan, X.; Zhang, W.; Zhao, C.; Yang, L.; Pan, R.; Chen, M.; Han, J.; Zhao, X.; et al. Utilization of a Wheat660K SNP array-derived high-density genetic map for high-resolution mapping of a major QTL for kernel number. Sci. Rep. 2017, 7, 3788. [CrossRef] [PubMed]

16. Winfield, M.O.; Allen, A.M.; Burridge, A.J.; Barker, G.L.; Benbow, H.R.; Wilkinson, P.A.; Coghill, J.; Waterfall, C.; Davassi, A.; Scopes, G.; et al. High-density SNP genotyping array for hexaploid wheat and its secondary and tertiary gene pool. Plant Biotechnol. J. 2016, 14, 1195-1206. [CrossRef]

17. Wang, S.; Wong, D.; Forrest, K.; Allen, A.; Chao, S.; Huang, B.E.; Maccaferri, M.; Salvi, S.; Milner, S.G.; Cattivelli, L.; et al Characterization of polyploid wheat genomic diversity using a high-density 90,000 single nucleotide polymorphism array. Plant Biotechnol. J. 2014, 12, 787-796. [CrossRef]

18. Cavanagh, C.R.; Chao, S.; Wang, S.; Huang, B.E.; Stephen, S.; Kiani, S.; Forrest, K.; Saintenac, C.; Brown-Guedira, G.L.; Akhunova, A.; et al. Genome-wide comparative diversity uncovers multiple targets of selection for improvement in hexaploid wheat landraces and cultivars. Proc. Natl. Acad. Sci. USA 2013, 110, 8057-8062. [CrossRef] [PubMed]

19. Wang, Z.; Liu, Y.; Shi, H.; Mo, H.; Wu, F.; Lin, Y.; Gao, S.; Wang, J.; Wei, Y.; Liu, C.; et al. Identification and validation of novel low-tiller number QTL in common wheat. Theor. Appl. Genet. 2016, 129, 603-612. [CrossRef]

20. Ma, L.; Bao, J.; Guo, L.; Zeng, D.; Li, X.; Ji, Z.; Xia, Y.; Yang, C.; Qian, Q. Quantitative trait loci for panicle layer uniformity identified in doubled haploid lines of rice in two environments. J. Integr. Plant Biol. 2009, 51, 818-824. [CrossRef]

21. Fang, F.; Ye, S.; Tang, J.; Bennett, M.J.; Liang, W. DWT1/DWL2 act together with OsPIP5K1 to regulate plant uniform growth in rice. New Phytol. 2020, 225, 1234-1246. [CrossRef]

22. Wang, W.; Li, G.; Zhao, J.; Chu, H.; Lin, W.; Zhang, D.; Wang, Z.; Liang, W. DWARF TILLER1, a WUSCHEL-related homeobox transcription factor, is required for tiller growth in rice. PLoS Genet. 2014, 10, e1004154. [CrossRef]

23. Zhao, C.; Zhang, N.; Wu, Y.; Sun, H.; Liu, C.; Fan, X.; Yan, X.; Xu, H.; Ji, J.; Cui, F. QTL for spike-layer uniformity and their influence on yield-related traits in wheat. BMC Genet. 2019, 20, 23. [CrossRef] [PubMed]

24. Malik, P.; Kumar, J.; Sharma, S.; Sharma, R.; Sharma, S. Multi-locus genome-wide association mapping for spike-related traits in bread wheat (Triticum aestivum L.). BMC Genom. 2021, 22, 597. [CrossRef] [PubMed] 
25. International Wheat Genome Sequencing Consortium (IWGSC). Shifting the limits in wheat research and breeding using a fully annotated reference genome. Science 2018, 361, 7191. [CrossRef]

26. Zhu, T.; Wang, L.; Rimbert, H.; Rodriguez, J.C.; Deal, K.R.; De Oliveira, R.; Choulet, F.; Keeble-Gagnère, G.; Tibbits, J.; Rogers, J.; et al. Optical maps refine the bread wheat Triticum aestivum cv. Chinese Spring genome assembly. Plant J. 2021, 107, 303-314. [CrossRef]

27. Jain, M.; Ghanashyam, C.; Bhattacharjee, A. Comprehensive expression analysis suggests overlapping and specific roles of rice glutathione S-transferase genes during development and stress responses. BMC Genom. 2010, 11, 73. [CrossRef]

28. Yao, W. Studies on inheritance of spike layer uniformity of wheat. Seed 2000, 3, 19-21.

29. $\mathrm{Hu}, \mathrm{Y}$. The difference of the spike-layer architecture and its relation to yield in winter wheat cultivars. Seed 2001, 119, 19-21.

30. Li, J.; Yan, Q. Studies on the relationship between the evenness degree and yield characters of hybrid early rice. Hunan Agric. Sci. 2005, 1, 14-17.

31. Zhao, D.H.; Yan, Q.Q.; Wang, J.R.; Wan, H.Q. Study on relationship between population uniformity and yield characters of late hybrid rice. Guangxi Agric. Sci. 2005, 36, 205-207.

32. Liu, Y.; Lin, Y.; Gao, S.; Li, Z.; Ma, J.; Deng, M.; Chen, G.; Wei, Y.; Zheng, Y. A genome-wide association study of 23 agronomic traits in Chinese wheat landraces. Plant J. 2017, 91, 861-873. [CrossRef] [PubMed]

33. Piepho, H.P.; Möhring, J.; Melchinger, A.E.; Büchse, A. BLUP for phenotypic selection in plant breeding and variety testing. Euphytica 2008, 161, 209-228. [CrossRef]

34. Smith, S.E.; Kuehl, R.O.; Ray, I.M.; Hui, R.; Soleri, D. Evaluation of simple methods for estimating broad-sense heritability in stands of randomly planted genotypes. Crop Sci. 1998, 38, 1125-1129. [CrossRef]

35. Li, H.; Hearne, S.; Banziger, M.; Li, Z.; Wang, J. Statistical properties of QTL linkage mapping in biparental genetic populations. Heredity 2010, 105, 257-267. [CrossRef]

36. Meng, L.; Li, H.; Zhang, L.; Wang, J. QTL IciMapping: Integrated software for genetic linkage map construction and quantitative trait locus mapping in biparental populations. Crop J. 2015, 3, 269-283. [CrossRef]

37. Deng, M.; Wu, F.; Zhou, W.; Li, J.; Shi, H.; Wang, Z.; Lin, Y.; Yang, X.; Wei, Y.; Zheng, Y.; et al. Mapping of QTL for total spikelet number per spike on chromosome 2D in wheat using a high-density genetic map. Genet. Mol. Biol. 2019, 42, 603-610. [CrossRef]

38. Rice, J.P.; Saccone, N.L.; Corbett, J. Model-based methods for linkage analysis. Adv. Genet. 2008, 60, 155-173. [CrossRef]

39. Lebrec, J.; Putter, H.; Houwelingen, J.C. Score test for detecting linkage to complex traits in selected samples. Genet Epidemiol 2004, 27, 97-108. [CrossRef] [PubMed]

40. Lin, Y.; Jiang, X.; Tao, Y.; Yang, X.; Wang, Z.; Wu, F.; Liu, S.; Li, C.; Deng, M.; Ma, J.; et al. Identification and validation of stable quantitative trait loci for grain filling rate in common wheat (Triticum aestivum L.). Theor. Appl. Genet. 2020, 133, $2377-2385$. [CrossRef] [PubMed]

41. Suzuki, Y.; Sekiya, T.; Hayashi, K. Allele-specific polymerase chain reaction: A method for amplification and sequence determination of a single component among a mixture of sequence variants. Anal. Biochem. 1991, 192, 82-84. [CrossRef]

42. Bu, D.; Luo, H.; Huo, P.; Wang, Z.; Zhang, S.; He, Z.; Wu, Y.; Zhao, L.; Liu, J.; Guo, J.; et al. KOBAS-i: Intelligent prioritization and exploratory visualization of biological functions for gene enrichment analysis. Nucleic. Acids Res. 2021, 49, W317-W325. [CrossRef] [PubMed]

43. Yao, W.; Li, G.; Yu, Y.; Ouyang, Y. FunRiceGenes dataset for comprehensive understanding and application of rice functional genes. Gigascience 2017, 7, 119. [CrossRef] [PubMed] 